\title{
Direct Observation of the Effects of Alloying Additions on Transformation Mechanisms in Emerging Steel Alloys with in situ TEM
}

\author{
M. Hartshorne*, E. Toby*, P. Uranga**, P. Novotny***, M. Schmidt**** and M.L. Taheri* \\ *Department of Materials Science, Lebow 344, Drexel University, Philadelphia, PA 19104 \\ **CEIT and TECNUN (University of Navarra), San Sebastian, Spain \\ *** Tool and Alloy R\&D, Carpenter Technology Corporation, Reading, PA 19601 \\ **** Stainless Steel Alloy R\&D, Carpenter Technology Corporation, Reading, PA 19601
}

Thermomechanical processing is a technique used to control the microstructure of steel, however steels which have are exposed to corrosive environments, high temperatures and dynamic loads will show evolutions in the microstructure from the initial product. The behavior of steel in moderate to high temperatures is especially relevant in applications such as internal combustion engines and nuclear power plants due to the long periods of time spent at elevated temperatures $[1,2]$. The segregation and precipitation of solutes in complex steel alloys can be studied in situ using a variety of high resolution microscopy methods in order to provide a more detailed understanding of these mechanisms.

Uranga and colleagues are currently conducting a research project to develop microalloyed steels. The segregation and precipitation of carbon and microalloying elements during and after phase transformations in these steels can be better understood by conducting and analyzing experiments in situ. Increased demand for thin slab direct rolled (TSRD) steels, due to improvements in the mechanical properties of these steels, gives impetus to the quantification of the wide range of microstructures exhibited by TSRD products [3]. Novotny and colleagues at Carpenter Technology Corporation have recently developed a line of steel alloys that exhibit a combination of high toughness and ductility while retaining high hardness and strength [4]. An analysis of the effect of solute additions during alloy processing is required to understand the evolution of the microstructure and its influence on the properties of these alloys.

We will analyze samples of steel from CEIT and Carpenter Technology Corporation utilizing a variety of methods. The primary experimental technique will be in situ microstructural evolution using in-TEM annealing coupled with energy dispersive spectroscopy. Electron Backscattered Diffraction (EBSD) will be employed to observe grain orientation, while techniques such as local electrode atom probe (LEAP) will be used to assess solute and precipitate location and chemistry. The data attained throughout this project will be used to develop a detailed view of segregation and precipitation of alloying elements in these steels when they are subjected to thermal treatments. This knowledge will provide a basis for improving processing techniques and an understanding of the behavior of the microstructure of these steels in high temperature applications.

References

[1] Nobuhiro Fujita, HKDH Bhadeshia and Masao Kikuchi. Modelling Simul. Mater. Sci. Eng. 12 (2004) 273. 
[2] B. Weiss and R. Stickler. Metallurgical Transactions 3 (1972) 851.

[3] P. Uranga, J. Ganzarain, D. Jorge-Badiola and J.M. Rodriguez-Ibabe. (2008) 1325.

[4] Carpenter Technology, 2009.

[5] This work is supported by Carpenter Technology Corporation and Drexel University. 